\title{
Developmental and neurological progress of preterm infants with intraventricular haemorrhage and ventricular dilatation
}

\author{
PENELOPE PALMER, LILLY M S DUBOWITZ, M I LEVENE, AND V DUBOWITZ \\ Department of Paediatrics and Neonatal Medicine, Institute of Child Health, Hammersmith Hospital, London
}

SUMmARY A prospective neurological and developmental assessment was completed at ages 6, 9, and 12 months on 39 preterm infants under 34 weeks' gestation. In the newborn period each infant had an assessment of gestation and sequential neurological and ultrasound examinations and was placed in one of three groups: intraventricular haemorrhage (IVH) $(n=14)$, IVH followed by ventricular dilatation $(n=11)$, and control infants with no evidence of IVH $(n=14)$. When corrected for prematurity the Griffiths's developmental quotients (DQs) were normal at 6, 9, and 12 months for every infant except one aged 12 months. In contrast, the uncorrected DQs at 12 months were under 80 in only one of the 14 preterm infants without haemorrhage, compared with 2 of the 14 with IVH, and with 7 of the 9 with IVH and dilatation. There was also a higher incidence of neurological abnormality at each follow-up age in the infants with IVH plus ventricular dilatation, compared with those with IVH alone, or with infants without IVH. Similar differences were also demonstrated in 5 milestones reflecting gross motor, fine motor, and social or verbal development in the three groups at 6,9, and 12 months. The neurological and developmental deficits seemed to relate more closely to the presence of post-haemorrhagic ventricular dilatation than to the size of the initial haemorrhage itself. These results may have important implications for therapeutic intervention in the management of newborn infants with IVH and ventricular dilatation.

Germinal layer haemorrhage and intraventricular haemorrhage (IVH) occur in about half of very low birthweight or preterm infants. ${ }^{1-3}$ The majority survive and it is clearly important to determine whether their early development differs from that of preterm infants without IVH and whether particular neurological abnormality, sensory deficit, or developmental delay is associated with germinal layer haemorrhage and IVH. The development of ultrasound techniques during the last few years has made possible not only the diagnosis of IVH and germinal layer haemorrhage at the cotside but also serial monitoring of the evolution and resolution of the haemorrhage and changes in ventricular size. ${ }^{45}$ The present study compares the early development up to age one year of a series of infants with germinal layer haemorrhage or IVH with a control group of preterm infants without haemorrhage.

\section{Material and methods}

The 39 infants in the study were all admitted to the neonatal unit at Hammersmith Hospital between November 1979 and August 1980. Twenty-five had germinal layer haemorrhage or IVH (which we colloquially refer to as IVH) and 11 of these developed subsequent ventricular dilatation. The 14 control infants were preterm infants of similar gestation, who had no ultrasound evidence of IVH or neurological abnormality on detailed clinical assessment, who had been chosen for comparative follow-up at the time of discharge from the neonatal unit.

Within 36 hours of admission each infant had an ultrasound scan, ${ }^{2}$ an assessment of gestation, ${ }^{6}$ and a detailed neurological examination. ${ }^{7}$ In infants below 31 weeks' gestation or those receiving intensive care, scans were performed daily for the first 2 weeks.

All scans were done by one experienced observer (M I L), using a Kranzbühler ADR real-time linear array machine. All infants had axial plane scans through the temporoparietal region using a $5 \mathrm{MHz}$ transducer and IVH was diagnosed if echoes were 
seen in the region of the lateral ventricle. In 27 of the 39 infants a transfontanelle scan was also performed using a $7 \mathrm{MHz}$ transducer to diagnose germinal layer haemorrhage by the presence of dense subependymal echoes in the region of the caudate nucleus.

The haemorrhage was graded as follows ${ }^{8}$ :

Grade I-Small haemorrhage, originating in the region of the germinal matrix with no inferior or lateral extension beyond the lateral-most border of the lateral ventricle.

Grade II-Moderate haemorrhage with some downwards extension into the basal nuclei on at least one side, or involvement of the caudate nucleus to the region of the genu of the lateral ventricle posteriorly on parasagittal scans.

Grade III-Large haemorrhage, with any degree of extension laterally or superolaterally into the cerebral parenchyma.

Ventricular dilatation was diagnosed if the ventricular index (the distance between the lateral ventricle and the falx on an axial scan through the temporoparietal region) showed an increase from normal value to above the 97 th centile for gestational age, ${ }^{4}$ or if the distance from the roof to the floor of the lateral ventricle exceeded $6 \mathrm{~mm} .^{5}$ These measurements were made at the body of the lateral ventricle.

The ultrasound findings were not available to the attending clinical staff on the neonatal unit or to the observers who carried out the follow-up assessments.

The babies were reviewed at 6,9 , and 12 months from birth. The follow-up examination consisted of a detailed neurological examination based on the schemes of Touwen ${ }^{9}$ and Ounsted (1978, personal communication) and an assessment of development using the Griffiths's scale. ${ }^{10}$ Infants in whom abnormal vision or hearing was suspected were referred for specialist ophthalmological or audiometric assessment.

The range in birthweight and gestational age, the sex distribution, and the incidence of major complications of prematurity, other than neurological, in the three groups of infants are shown in Table 1. Major complications-such as severe respiratory distress syndrome, recurrent apnoea, proved sepsis, or necrotising enterocolitis-occurred in all the infants in the group with IVH and dilated ventricles, in over half of those with IVH alone, and in about one-third of the control infants.

The ultrasound data are summarised in Table 2. In the group with IVH only, two infants had a grade III haemorrhage and in the remaining twelve the haemorrhage was grade I. Of the infants with
Table 1 Clinical details of infants in the study

\begin{tabular}{lccc}
\hline & $\begin{array}{l}\text { IVH+ventricular } \\
\text { dilatation } \\
(n=11)\end{array}$ & $\begin{array}{l}\text { IVH only } \\
(n=14)\end{array}$ & $\begin{array}{c}\text { No IVH } \\
(n=14)\end{array}$ \\
\hline $\begin{array}{l}\text { Birthweights (g) } \\
\begin{array}{l}\text { Gestational age } \\
\text { (weeks) }\end{array}\end{array}$ & $\begin{array}{c}920-2500 \\
27-34\end{array}$ & $\begin{array}{c}790-1980 \\
28-33\end{array}$ & $\begin{array}{c}1130-2220 \\
29-34\end{array}$ \\
$\begin{array}{c}\text { Small for } \\
\text { gestational age } \\
(<10 \text { centile) }\end{array}$ & 0 & 3 & 3 \\
$\begin{array}{l}\text { Gender } \\
\text { Major } \\
\text { complications }\end{array}$ & 5 boys, 6 girls & 6 boys, 8 girls & $\begin{array}{c}8 \text { boys, } 6 \text { girls } \\
5\end{array}$ \\
\hline
\end{tabular}

Table 2 Correlation of ventricular dilatation with size of intraventricular haemorrhage

\begin{tabular}{llll}
\hline & \multicolumn{2}{l}{ Grade } & \\
\cline { 2 - 4 } Size of IVH & $I$ & $I I$ & $I I I$ \\
\hline Number of cases & 1 & 5 & 5 \\
Ventricular dilatation & & & \\
$\quad$ Transient (<30 days) & 0 & 3 & 1 \\
Persistent (80-270 days) & 1 & 1 & 3 \\
Shunted & 0 & 1 & 0 \\
Cerebral atrophy & 0 & 0 & 1 \\
\hline
\end{tabular}

IVH who developed ventricular dilatation the haemorrhage was grade I in one, grade II in five, and grade III in five. In four infants the dilatation was transient and resolved without treatment within a month of onset. In five it was persistent but nonprogressive and was associated with normal head growth. One infant had progressive ventricular dilatation, an increasing head circumference, and a ventriculo-peritoneal shunt. One infant had irregular and asymmetrical ventricular dilatation and poor head growth, assumed to be due to cerebral atrophy. This infant subsequently died and cerebral atrophy was confirmed at necropsy.

\section{Results}

All but 2 of the infants have been followed up until aged one year. One infant, with ventricular dilatation and cerebral atrophy who died at 4 months, already had overt clinical evidence of spastic quadriplegia. One infant with a large haemorrhage (grade III) and persistent ventricular dilatation returned with his parents to Holland. A neurological protocol was completed by his attending paediatrician but he did not have a Griffiths's developmental examination.

Neurological abnormalities. The neurological abnormalities seen in the three groups of infants at 6-, 9-, and 12-month examinations are shown in Table 3. 
Table 3 Neurological signs at follow-up examination at 6,9 , and 12 months of age

\begin{tabular}{|c|c|c|c|}
\hline & $\begin{array}{l}\text { No IVH } \\
(n=14)\end{array}$ & $\begin{array}{l}\text { IVH only } \\
(n=14)\end{array}$ & $\begin{array}{l}\text { IVH+ } \\
\text { ventricular } \\
\text { dilatation } \\
(n=11)\end{array}$ \\
\hline $\begin{array}{l}\text { 6-month examination } \\
\text { (Number examined) }\end{array}$ & (14) & $(12)$ & (9) \\
\hline Asymmetry & 0 & 0 & 3 \\
\hline Hypertonia & 0 & 2 & 3 \\
\hline Hypotonia & 0 & 1 & 0 \\
\hline Head lag & 2 & 4 & 3 \\
\hline Fisting & 0 & 0 & 2 \\
\hline $\begin{array}{l}\text { Suspected abnormal hearing } \\
\text { or vision }\end{array}$ & $\mathbf{0}$ & 4 & 4 \\
\hline No abnormal signs & 12 & 4 & 0 \\
\hline $\begin{array}{l}\text { 9-month examination } \\
\text { (Number examined) }\end{array}$ & (12) & (11) & (8) \\
\hline Asymmetry & 1 & 1 & 1 \\
\hline Hypertonia & 1 & 4 & 4 \\
\hline Hypotonia & 0 & 2 & 0 \\
\hline Visual or hearing defect & 0 & 3 & 3 \\
\hline No abnormal signs & 10 & 4 & 1 \\
\hline $\begin{array}{l}\text { 12-month examination } \\
\text { (Number examined) }\end{array}$ & (14) & (14) & (10) \\
\hline Asymmetry & 1 & 2 & 5 \\
\hline Hypertonia & 0 & 2 & 1 \\
\hline Hypotonia & 0 & 2 & 2 \\
\hline Fine motor dysfunction & 0 & 1 & 1 \\
\hline Defective vision & 0 & 0 & 1 \\
\hline Strabismus & 0 & 1 & 2 \\
\hline Hearing defect & 0 & $2^{*}$ & 1 \\
\hline No abnormal signs & 13 & 6 & 1 \\
\hline
\end{tabular}

Of the fourteen infants with no IVH, two showed head lag at 6 months, two showed minor neurological abnormalities at 9 months, and one had minor asymmetry at 1 year. None had major defects of vision or hearing.

The IVH group showed neurological abnormalities in eight of the twelve examined at 6 months, in seven of eleven assessed at 9 months, and in eight of fourteen examined at 12 months. At one year two had significant hypotonia and were not weightbearing; one had marked impairment of fine movements; and five had such minor neurological abnormalities as mild hypertonia $(n=2)$, mild asymmetry $(n=2)$, and strabismus $(n=1)$. Two infants had conductive hearing defects, thought to be due to bilateral suppurative otitis media.

In the ten surviving infants with IVH and ventricular dilatation, neurological abnormalities were found in all eight infants examined at 6 months, in seven of the eight at 9 months, and in nine of the ten examined at 12 months. At 12 months five babies showed asymmetry (including hemiplegia in two), one hypertonia, and three lower limb hypotonia with absent weight-bearing. One baby had fine motor incoordination and one defective vision. Hearing defect was present in one infant, who had profound sensorineural deafness.

The incidence of neurological abnormality was greater in the IVH group than in the controls at 6 9 , and 12 months but did not reach statistical significance. However, the differences between the group with IVH and ventricular dilatation and the control group reached statistical significance at each age tested $(P<0.01$ at 6 months; $P<0.05$ at 9 months; and $P<0.01$ at 12 months (Fisher's exact test)). The incidence of major handicap at 1 year was also significantly greater in the IVH+dilatation group ( $\mathrm{P}<0.01$; Fisher's exact test).

Developmental assessment. The developmental quotients on the Griffiths's scale for the three groups of infants at 6, 9, and 12 months from birth are shown in Figs 1 and 2.

Fig. 1 shows the quotients corrected for prematurity (that is the infant's gestational age at birth is subtracted from 40 and this number is subtracted

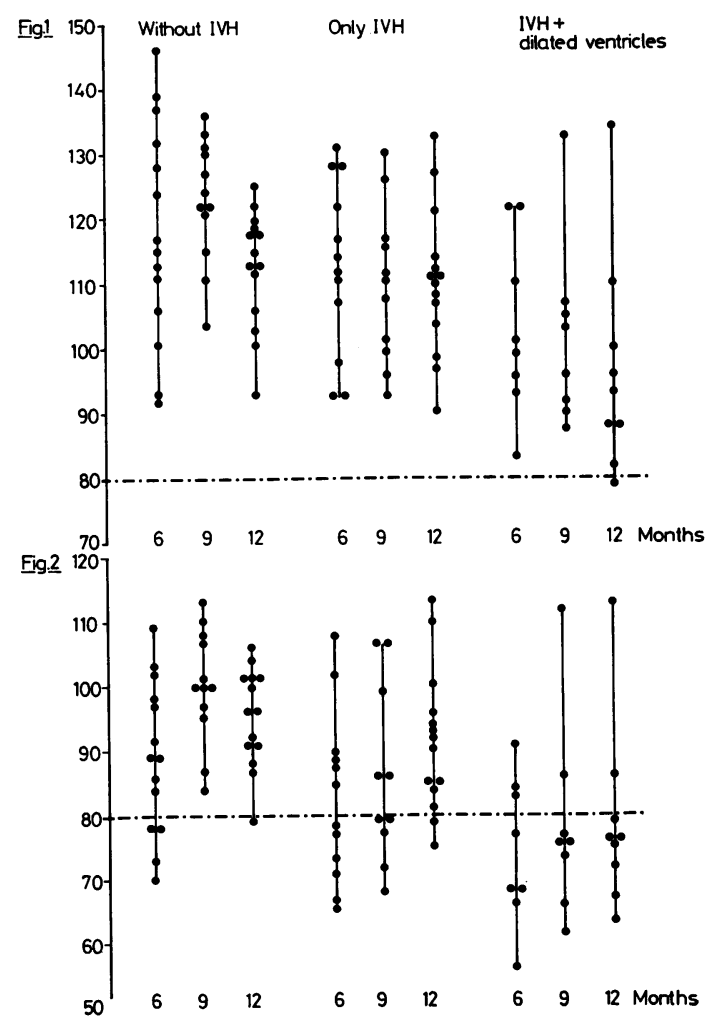

Fig. 1 Developmental quotients on the Griffiths's scale of the three groups of infants at 6, 9, and 12 months' chronological age, corrected for prematurity.

Fig. 2 Developmental quotients on the Griffiths's scale of the three groups of infants at 6, 9, and 12 months' chronological age, uncorrected for prematurity. 
from the actual age in weeks at testing). The corrected DQ was normal at 6,9, and 12 months in all but one infant in the IVH and ventricular dilatation group at 12 months.

The uncorrected developmental quotients (Fig. 2) give an indication of developmental catch-up (or deterioration) in the three groups of infants. In the infants with no IVH, the DQ was below 80 in four of the fourteen tested at 6 months but in only one of fourteen by 12 months. In the group with IVH the DQ was below 80 in six of the twelve infants at 6 months but in only two of fourteen at 12 months. In the group with IVH and ventricular dilatation, five of the eight tested were below 80 at 6 months, compared with seven of the nine tested at 12 months.

The differences in DQ in the IVH+dilatation group compared with the control group were significant at all ages tested for both the corrected and uncorrected values (at 6 months $P<0.04$ and $<0.01$; at 9 months $P<0.01$ and $<0.05$; at 12 months $\mathbf{P}<0.05$ and $<0.01$ respectively (Student's $t$ test)).

Clinical assessment. In order to see if a simple clinical assessment could identify the differences between the groups noted above, we selected for analysis from our data 5 items appropriate to each age group tested $(6,9$, and 12 months). These items are part of the paediatrician's clinical armamentarium, and cover gross motor, fine motor, and social or verbal development. The proportion of infants in each of the three groups achieving these milestones at 6, 9, and 12 months is shown in Figs 3, 4, and 5.

It will be noted that for each item tested at each age period the infants without IVH did better than those with IVH, who in turn did better than those with IVH and dilated ventricles. Most of the babies with dilated ventricles showed considerable develop-

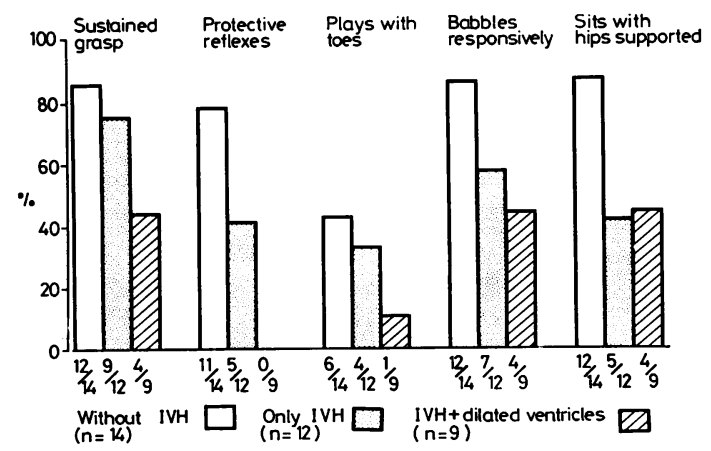

Fig. 3 Proportion of infants in each of the three groups achieving various milestones at 6 months.

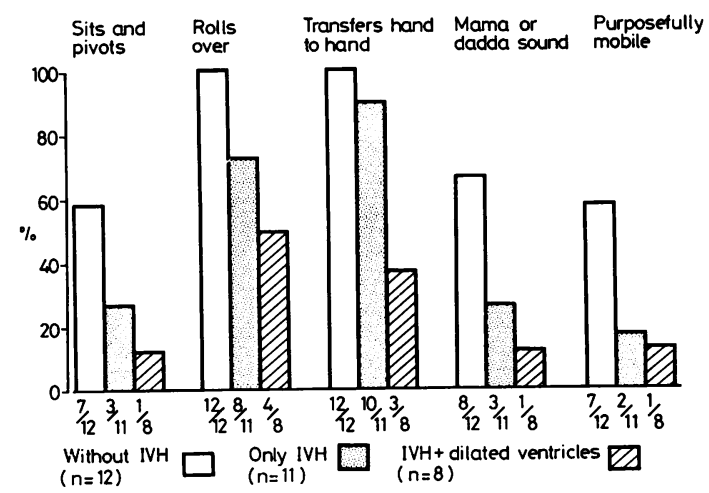

Fig. 4 Proportion of infants in each of the three groups achieving various milestones at 9 months.

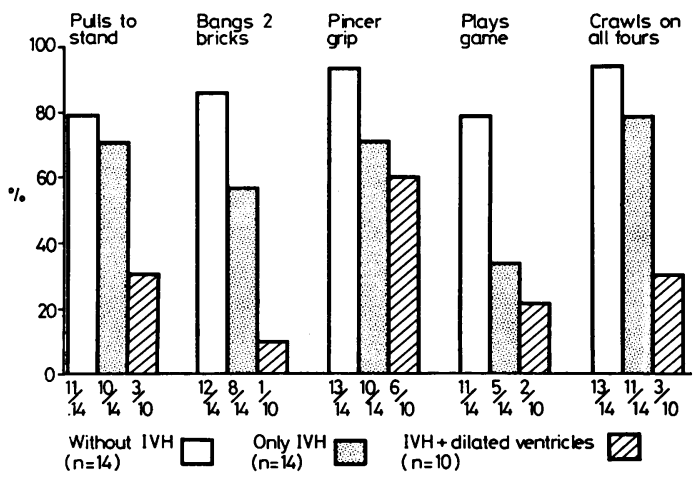

Fig. 5 Proportion of infants in each of the three groups achieving various milestones at 12 months.

mental delay compared with the babies without IVH and were particularly delayed in items requiring two-handed manipulation at both 9 months (transferring hand to hand) and 12 months (banging two bricks together).

Overall status at one year. There were no infants with major handicaps in the non-IVH or IVH groups, whereas 6 of the 10 infants with IVH plus ventricular dilatation had major handicaps at 1 year (Table 4). These included developmental delay, profound sensorineural deafness, visual impairment, hemiplegia, and pronounced fine motor incoordination.

\section{Discussion}

A fairly good outcome for the survivors of small germinal layer haemorrhages has been reported 
Table 4 Developmental and neurological data on infants with intraventricular haemorrhage plus ventricular dilatation

\begin{tabular}{|c|c|c|c|c|c|}
\hline $\begin{array}{l}\text { Gestation } \\
\text { (weeks) }\end{array}$ & $\begin{array}{l}\text { Size of } \\
\text { IVH }\end{array}$ & $\begin{array}{l}\text { Ventricular } \\
\text { dilatation }\end{array}$ & Neurological status & $\begin{array}{l}D Q \text { (Griffiths) } \\
\text { corrected / } \\
\text { uncorrected }\end{array}$ & Current status \\
\hline 27 & Medium & Transient & Hypotonia. Not weight-bearing. Poor sitting & $96 / 72$ & Suspect \\
\hline 28 & Large & Transient & Asymmetry (severe). Hemiplegia. Hypertonia & $100 / 76$ & Handicapped* \\
\hline 29 & Medium & Transient & Asymmetry (mild). Deafness. Visual defect & $93 / 75$ & Handicapped \\
\hline 29 & Large & Persistent & Asymmetry (severe). Hypertonia & & Handicapped \\
\hline 29 & Medium & Persistent & Asymmetry (mild) & $110 / 86$ & Normal \\
\hline 30 & Large & Persistent & Asymmetry (severe). Hemiplegia. Hypertonia & $78 / 65$ & Handicapped \\
\hline 30 & Small & Persistent & Hypotonia. No weight-bearing. Poor sitting & $82 / 67$ & Handicapped \\
\hline 32 & Large & Persistent & Hypotonia. Poor weight bearing. Unable to get to sitting. Squint & $88 / 75$ & Suspect \\
\hline 32 & Medium & Transient & None & $133 / 112$ & Normal \\
\hline 34 & Medium & Shunt & Lower limb hypertonia. Very clumsy & $89 / 79$ & Handicapped \\
\hline 34 & Large & Cerebral atrophy & Shunt. Died at $4 / 12$ & & \\
\hline
\end{tabular}

*Abnormal neurological signs interfering with function, deafness, DQ uncorrected $<70$.

by several authors. ${ }^{311} 12$ The present study has shown that infants with ventricular dilatation after IVH have a high incidence of neurological handicap, while the early development of infants with IVH but without dilatation is not very different from that of babies of similar gestation without haemorrhage and with optimal neurological status, despite more frequent minor neurological abnormalities.

It is of interest to compare our results with those of Thorburn et al. ${ }^{3}$ who prospectively studied a similar group of preterm infants. They found that the size of the initial haemorrhage was the major determinant of outcome; those infants who survived large IVH had the most severe degrees of neurological handicap. While it cannot be disputed that the greater degree of intracerebral bleeding must in general terms be associated with more severe disruption, and this might be expected to be more likely to lead to handicap, our findings suggest that other factors may influence the poor outcome at age one year. The infants who developed either transient or persistent ventricular dilatation after IVH showed a high incidence $(60 \%)$ of serious neurological abnormalities and developmental delay, irrespective of the size of the preceding IVH. Dilatation of cerebral ventricles after IVH may reflect a general cerebral abnormality leading to compression of the cerebral mantle, possibly with a resultant reduction in cell numbers. ${ }^{13}$

It is noteworthy that each of the 2 infants with large IVH, but without ventricular dilatation, was free from major handicap at one year, while 4 of the 5 infants with large IVH and dilated ventricles had major handicaps. The one infant with a small IVH and subsequent ventricular dilatation showed considerable developmental delay at one year, but all 12 infants with small IVH without dilatation were free from major neurological sequelae.

It seems probable that an ill preterm infant at risk of IVH will also be susceptible to ischaemic cerebral insults through the mechanisms described by Pape and Wigglesworth. ${ }^{14}$ Unfortunately, ischaemic lesions cannot be recognised in immature infants with currently available cerebral imaging techniques, and consequently the handicap will be attributed to IVH. The possibility exists that infants in this study with persistent ventricular dilatation have some degree of ischaemic cerebral atrophy, despite normal head growth. We believe however, that cerebral atrophy can often be recognised by specific abnormalities seen on ultrasound scans, and only one infant in this study appeared to have these findings. This infant subsequently died and atrophy was confirmed at necropsy. In 4 infants with transient ventricular dilatation, 2 were found to be handicapped at one year. As the ventricular size in these infants spontaneously returned to normal, significant cerebral atrophy could not have been present in them.

It is of interest that only one infant in our entire series had a development quotient corrected for prematurity which fell below the dividing line of 'normality' (80). However, inspection of the corrected DQs in the three groups of infants shows that, although all three are within the normal range, the actual distribution of the infants within each group is very different. On the other hand, the uncorrected DQs showed that 13 of the 14 control infants were within the normal range by one year, whereas 7 of the 9 infants with ventricular dilatation were still below. This stresses the potential danger of drawing conclusions on the developmental progress of preterm infants with a particular problem, or treated by a trial regimen, on the basis of developmental quotients corrected for the age deficit of prematurity, without comparison with the range of DQs in a control population of similar gestation.

We would advise the use of uncorrected DQs as a better screen for persisting deficit rather than the corrected DQs. This is also reflected in the pronounced differences in achievement of individual 
developmental milestones between the three groups of infants in our study at the 6-, 9-, and 12-month follow-up. Although most of the infants with IVH but without dilatation appeared to become normal by a year, long-term follow-up will be necessary to see if they are at particular risk of learning, behavioural, and other disabilities in later childhood.

As the most severe sequelae seemed to be associated with the presence of ventricular dilatation, one has to ask whether the compressive effects of ventricular dilatation may cause severe and permanent neurological damage independently of the antecedent factors, which in themselves could cause the neurological sequelae as well as the dilatation, resulting in the ventricular dilatation. This raises the question of whether early intervention aimed directly at controlling the ventricular dilatation may have a beneficial effect on the neurological and developmental outcome. We are currently conducting a randomised controlled clinical trial to compare ventricular decompression by regular lumbar and ventricular fluid taps against our current policy of non-intervention unless rapid head growth occurs.

We thank the neonatal nursing staff for help, Professor M J R Healy for the statistical analyses, and Val Chalk for secretarial assistance.

Financial support was provided by the Wellcome Trust and the Medical Research Council.

\section{References}

1 Papile L-A, Burstein J, Burstein R, Koffler H. Incidence and evolution of subependymal and intraventricular hemorrhage: a study of infants with birth weights less than 1,500 gm. J Pediatr 1978; 92: 529-34.

2 Levene M I, Wigglesworth J S, Dubowitz V. Cerebral structure and intraventricular haemorrhage in the neonate: a real-time ultrasound study. Arch Dis Child 1981 ; 56: 416-24.
3 Thorburn R J, Lipscomb A P, Stewart A L, Reynolds E O R, Hope P L, Pape K E. Prediction of death and major handicap in very preterm infants by brain ultrasound. Lancet 1981; i: 1119-21.

4 Levene M I, Starte D R. A longitudinal study of posthaemorrhagic ventricular dilatation in the newborn. Arch Dis Child 1981 ; 56: 905-10.

5 Levene M I. Measurement of the growth of the lateral ventricles in preterm infants with real-time ultrasound. Arch Dis Child 1981; 56: 900-4.

6 Dubowitz L M S, Dubowitz V, Goldberg C. Clinical assessment of gestational age in the newborn infant. $J$ Pediatr 1970; 77: 1-10.

7 Dubowitz L M S, Dubowitz V. The neurological assessment of the preterm and full-term newborn infant. Clinics in Developmental Medicine No 79. London: Heinemann, 1981.

8 Levene M I, Fawer C-L, Lamont R F. Risk factors in the development of intraventricular haemorrhage in the preterm neonate. Arch Dis Child 1982; 57: 410-7.

9 Touwen B. Neurological development in infancy. Clinics in Developmental Medicine No 58. London: Heinemann, 1976.

10 Griffiths R. The abilities of babies: a studv in mental measurement. Amersham: Association for Research in Infant and Child Development, 1976.

11 Krishnamoorthy K S, Shannon D C, DeLong G R, Todres I D, Davis $K$ R. Neurologic sequelae in the survivors of neonatal intraventricular hemorrhage. Pediatrics 1979; 64: 233-7.

12 Papile L-A, Munsick G, Weaver N, Pecha S. Cerebral intraventricular hemorrhage $(\mathrm{CVH})$ in infants $<1500$ gms: developmental follow-up at one year (abstract). Pediatr Res 1979; 13: 528.

13 Weller $\mathrm{R}$ O, Shulman K. Infantile hydrocephalus: clinical, histological, and ultrastructural study of brain damage. $J$ Neurosurg 1972; 36: 255-65.

14 Pape K E, Wigglesworth J S. Haemorrhage, ischaemia, and the perinatal brain. Clinics in Developmental Medicine No 69/70. London: Heinemann, 1979.

Correspondence to Professor V Dubowitz, Department of Paediatrics and Neonatal Medicine, Hammersmith Hospital, Du Cane Road, London W12 0HS.

Received 1 June 1982 